nephron

Practice
Nephron 2018;140:129-133

DOI: 10.1159/000491440
Received: May 24, 2018

Accepted after revision: June 21, 2018

Published online: July 10, 2018

\title{
Proteomic Analysis for Identification of Biomarkers that Predict Severe Acute Kidney Injury
}

\author{
John M. Arthur ${ }^{a, b}$ Nithin Karakalaa, ${ }^{a}$ Ricky D. Edmondson ${ }^{a}$ \\ ${ }^{a}$ Department of Medicine, Division of Nephrology, University of Arkansas for Medical Sciences, \\ Little Rock, AR, USA; ${ }^{b}$ Central Arkansas Veterans Healthcare System, Little Rock, AR, USA
}

\section{Keywords}

Mass spectrometry · Proteomics · Biomarkers ·

Acute kidney injury · Urine

\begin{abstract}
The search for acute kidney injury (AKI) biomarkers has identified a number of urine proteins that can be used to predict the presence of AKI but has struggled to identify proteins that are prognostic for severe AKI. In this review, we discuss 2 currently available biomarkers and the designs of the studies in which they were identified and relate this to the AKI characteristics they predict clinically. We discuss recent advances in mass spectrometry and sample preparation, which have improved the ability to identify low abundance proteins as well as the ability to characterize more of the protein by mass spectrometry. We show how these changes can lead to a deeper and more thorough analysis of the urine proteome. Finally, we highlight 2 important issues that can help in the identification of these biomarkers, appropriate study design and adequate technical characteristics in the analysis.

(c) 2018 S. Karger AG, Basel
\end{abstract}

The search for biomarkers that predict progression to severe acute kidney injury (AKI) has produced biomarkers that predict the presence of AKI and the development of moderate AKI but has had limited success in identifying biomarkers that differentiate severe AKI from less severe forms. For instance, the concentration of urinary neutrophil gelatinase associated lipocalin (NGAL) has been used to predict AKI including its use in 55 interventional studies of AKI listed in ClinicalTrials.gov. However, in one of the original studies to evaluate its ability to predict severe AKI [1] only one-third of patients in the highest quintile had progression of a stage of AKI and $39 \%$ of those that progressed were not in the highest quintile. The area under the receiver operator curve was 0.58 . The product of urine concentrations of insulin-like

Contribution from the AKI and CRRT 2018 Symposium at the 23rd International Conference on Advances in Critical Care Nephrology, Manchester Grand Hyatt, San Diego, CA, USA, February 26-March 1, 2018. This symposium was supported in part by the NIDDK funded University of Alabama at Birmingham-University of California San Diego O'Brien Center for Acute Kidney Injury Research (P30DK079337).

\section{KARGER}

(c) 2018 S. Karger AG, Basel

E-Mail karger@karger.com

www.karger.com/nef
Dr. John M. Arthur

Department of Medicine, Division of Nephrology

University of Arkansas for Medical Sciences

4301 West Markham St, Slot 501, Little Rock, AR 72205 (USA)

E-Mail jmarthur@uams.edu 
growth factor binding protein 7 (IGFBP7) and metalloprotease inhibitor 2 (TIMP-2) is commercially available as an AKI biomarker. Although the IGFBP7 $\times$ TIMP-2 has good performance to predict the development of AKI, its ability to predict severe AKI is not as good with an area under the receiver operator curve of 0.69 for the prediction of progression to AKI stage 3 [2]. The inability of currently available biomarkers to differentiate severe AKI from more moderate AKI is a major limitation in the field. If biomarkers are going to be used to enrich enrollment in clinical trials, they will need to better identify patients who are likely to develop severe AKI.

Understanding how currently available biomarkers were developed is helpful in designing future studies to identify biomarkers that predict the progression to severe AKI. NGAL was identified using microarray analysis of renal tissue after unilateral renal ischemia in mice $[3,4]$. The studies were designed to identify RNA signals that increase in response to AKI. Because of this design, NGAL is good at predicting the development of AKI although not so good at predicting progression to severe AKI. The use of IGFBP7 and TIMP-2 as AKI biomarkers was first published in the Sapphire study [5]. The discovery cohort compared 340 biomarkers in 522 subjects. The results in the discovery cohort were used to justify validation of the product of IGFBP7 and TIMP-2 in a cohort that included 1,028 subjects. The primary outcome was developing stage 2 or 3 AKI within $12 \mathrm{~h}$. The product of the 2 biomarkers had an area under curve of 0.80 to predict the combined outcome but of the 101 subjects that met the endpoint of stage 2 or $3 \mathrm{AKI}$, only 18 achieved stage 3 . The predominance of stage 2 AKI in this cohort is likely the reason that the IGFBP7 $\times$ TIMP2 biomarker does not discriminate well between stage 2 AKI and more severe AKI. Sample size is important to improve the robustness of biomarker studies. While the overall numbers of progressors and non progressors was good in this study, the number of subjects with severe AKI was limited. New studies are needed to identify biomarkers that can differentiate those who will progress to severe AKI from those with more moderate AKI. These studies should utilize human samples with no AKI or stage $1 \mathrm{AKI}$ at the time the samples were collected, contain sufficient numbers of patients in each cohort, and identify biomarkers that can predict the progression to severe AKI. It is best to compare subjects with discrete and extreme phenotypes in these studies, that is, compare subjects with no further progression after the sample was collected with those who progressed to severe AKI.
Protein mass spectrometry involves the ionization of peptides and separation of the peptides based on their mass to charge ratio $(\mathrm{m} / \mathrm{z})$. Recent increases in sensitivity, speed, and resolution of mass spectrometry techniques along with new protocols for sample preparation, improvements in electrospray technology, nanoflow separation, and prefractionation have dramatically improved the capabilities of mass spectrometry-based biomarker discovery over the last few years. Figure 1 shows the features that can be used to assess sensitivity, resolution, and speed. Sensitivity is the ability of the instrument to detect low abundance peptide ions. Sensitivity is demonstrated by the large range of counts (Abundance) between Figure 1a where a peptide from the highest abundance protein in the gel slice can be seen eluting at 21.35 min with an abundance of 2E10 counts and Figure 1c where a peptide from IGFBP1 at $\mathrm{m} / \mathrm{z}$ of 510.951 can be seen with an abundance of 1E6. The ability to observe peptides that differ in abundance of 4 orders of magnitude or more increases the number of peptides that can be included in the analysis. Importantly, in our studies, IGFBP1 is the 216th most abundant urine protein (based on precursor intensity) in patients with AKI of the 2,428 proteins observed so many peptides present at much lower abundance than this peptide from IGFBP1 were identified. Scan speed is the number of scans that can be done per second. The importance of scan speed can be observed from Figure 1b, c. Although the spectrum in Figure 1b appears to have only a few peptides, when a small range of $\mathrm{m} / \mathrm{z}$ values in what appears to be a barren portion of the spectrum (zoomed in C) is observed, it can be seen that the spectrum is rich in peptide ions. Identification requires fragmentation and analysis of each of the peptide ions. Faster scan speed leads to the identification of more of the peptides in the spectrum. Resolution impacts the ability of the instrument to separate 2 peptides that are similar in $\mathrm{m} / \mathrm{z}$. High resolution allows the instrument to define a peptide as a narrow peak and separate it from its neighbors. In Figure 1d, 4 clearly resolved peaks can be seen at about $511.2 \mathrm{~m} / \mathrm{z}$ within 0.05 $\mathrm{m} / \mathrm{z}$ of each other. In a lower resolution instrument, these 4 discrete peptide ions would all appear as one peak, likely resulting in a missed identification. Higher resolution improves the ability to accurately measure the intensity of peptide peaks that have very similar masses. The refinement of technology since the release of the first orbitrap instrument in 2005 has had a large impact on the field of protein analysis and successive instruments have included improvements in ion fragmentation, changes to the ion trap, engineering enhancements to improve speed, sensitivity and resolution [6]. The addition of an 
Fig. 1. Relationship of Sensitivity, Resolution and Speed in Mass Spectrometry. a Base Peak Chromatogram showing elution time vs. total ion count of a single gel slice from urine from a patient with AKI. A peptide from the most abundant protein in the gel slice (alpha-1 microglobulin) is the major contributor to the abundant peak (2E10 counts) that elutes at $21.35 \mathrm{~min}$. The arrow represents the time point with relatively low abundance values at $16.78 \mathrm{~min}$ where spectra in subsequent panels were obtained. b The spectrum shows the full ion scan at $16.78 \mathrm{~min}$. Three peaks have an abundance of at least 2E8. A relatively quiet section of the spectrum between $\mathrm{m} / \mathrm{z} 502$ and 520 is highlighted, which is shown in the next panel. c The $\mathrm{m} / \mathrm{z}$ region between 502 and 520 demonstrates that many peptide ions can be seen in the spectrum. Three peptide ions at the arrows with the first at $\mathrm{m} / \mathrm{z} 510.951$ represent triply charged isotopes of a peptide from IGFBP1. d Increased magnification of a small range of the spectrum from $C$ showing high resolution of peptide ions with very similar masses. The arrows show the 3 isotopes of the IGFBP1 peptide ions.
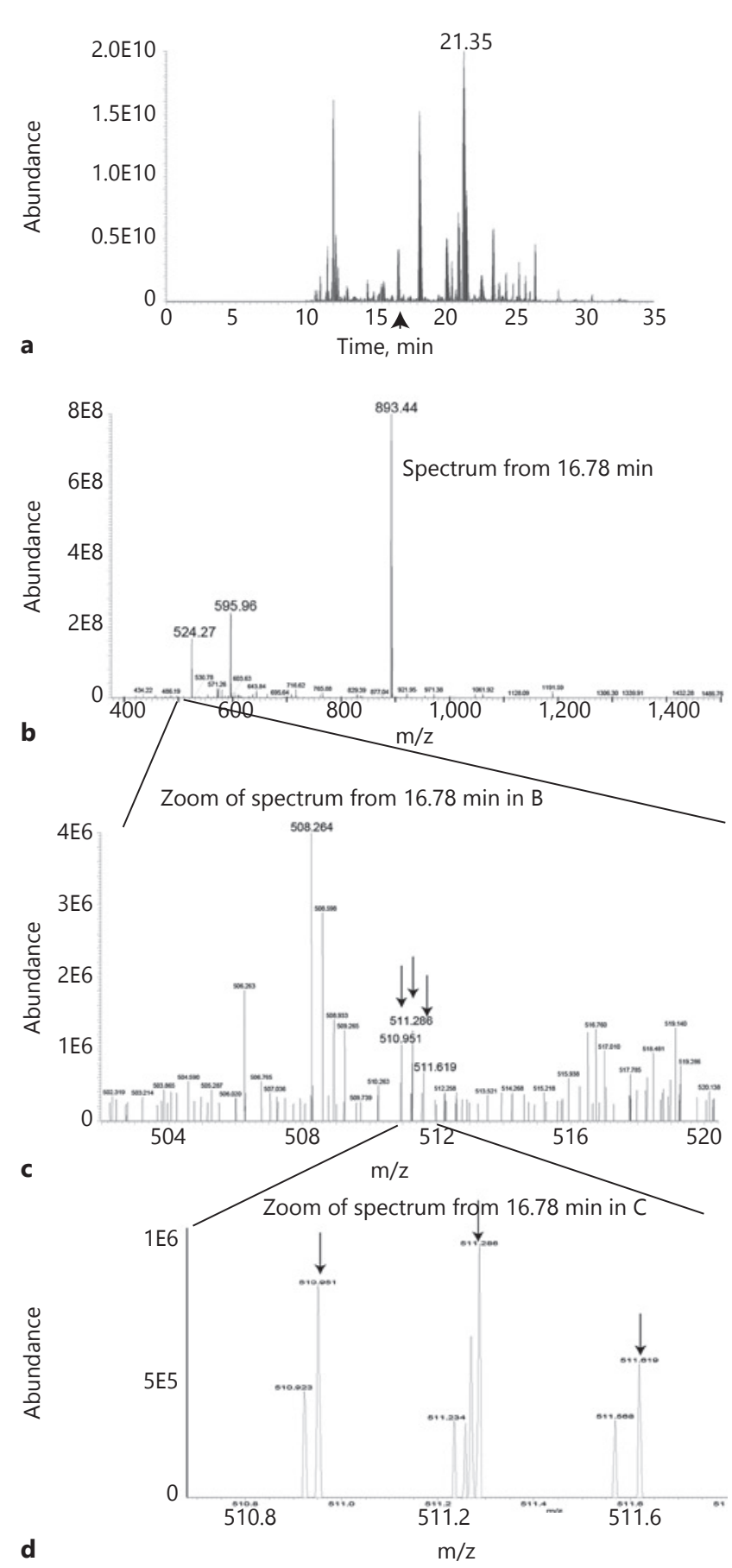

advanced quadrupole mass filter in the orbitrap Fusion and Lumos along with improvements in electronics and engineering has further increased the numbers of proteins and percentage of the protein observed as peptides (coverage).
As the technology of mass spectrometry has evolved, so have the techniques used for preparation and separation of proteins prior to injection of proteins. Filter digestion of samples (called Filter Assisted Sample Preparation) has led to shortening of sample preparation time with im- 
Table 1. Comparison of results from three proteomic analyses of urine

\begin{tabular}{llll}
\hline & LC-2013 & LC-2017 & GeLC-2018 \\
\hline Samples & 12 & 30 & 30 \\
Mass spectrometer & 5,600 & Fusion & Fusion \\
Instrument resolution & 30,000 & 500,000 & 500,000 \\
Digestion & In-solution & FASP & In-gel \\
Fractions & 7 & 1 & 24 \\
Proteins 1\% FDR & 359 & 2,428 & 3,629 \\
Proteins (2+ peptides) & 339 & 1,808 & 3,421 \\
Peptides & 4,866 & 13,691 & 44,722 \\
Median sequence coverage, \% & 15.4 & 14.3 & 31.0 \\
Median peptides per protein & 3 & 3 & 7 \\
Time for analysis (h) & 63 & 55 & 420 \\
\hline
\end{tabular}

proved proteome coverage [7]. Orthogonal separation techniques for prefractionation like off-line high-pH reversed phase chromatography with sample concatenation have improved sequence coverage and simplified sample processing [8]. In addition to data-dependent analysis of peptide ions, in which individual peptide ions are selected for fragmentation and identification, regions of the spectra containing multiple peptide ions can be fragmented and analyzed. The spectra are then compared to premade spectral libraries (from data-dependent analysis studies) to perform identification. This approach called data-independent analysis can result in more uniform identification of peptides. These and other improvements in sample preparation have worked synergistically with improvements in mass spectrometer technology to improve the ability to identify protein biomarkers in urine.

As an example of the differences that can be seen using more advanced instrumentation and sample preparation methods, we have compared the proteomic results from studies of urine samples that were analyzed in 2013 with samples from the same cohort that were analyzed by different methods in 2017 and 2018. The samples came from a cohort of patients collected by the SAKInet consortium. Both analyses used patients with stage $1 \mathrm{AKI}$ after cardiac surgery and compared patients who had no further progression to those who went on to develop severe AKI requiring renal replacement therapy. The first analysis (LC2013) used in-solution digestion, prefractionation into 7 fractions by solid phase extraction followed by reversed phase chromatography, and tandem mass spectrometry on an older instrument. Urinary angiotensinogen was identified as a prognostic biomarker in these studies [9]. The second cohort did not use prefractionation but used a newer mass spectrometer (Thermo Scientific orbitrap Fusion). The final study again used the Fusion, but one- dimensional polyacrylamide gel electrophoresis was done and each gel lane was sliced into 24 pieces that were individually analyzed. Table 1 shows the number of proteins and the characteristics of the protein coverage in each of the studies. Improvements between the 2013 and 2017 study were an increase in the number of samples analyzed, a change to digestion using the Filter Assisted Sample Preparation protocol, and the use of a mass spectrometer with greater sensitivity and resolution. This resulted in a large increase in the number of high confidence proteins observed $(359-2,428)$ with similar median protein sequence coverage. The change between the 2017 and 2018 studies was the use of prefractionation by gel separation. This analysis resulted in a further $50 \%$ increase in the number of proteins to 3,629 with a doubling in the median sequence coverage, which dramatically improves the confidence in identifying differentially abundant proteins. Further refinements, which may better elucidate high-quality biomarkers, are focusing on post-translational modifications or specific components of fluids such as exosomes.

In summary, the identification of novel urinary biomarkers that can predict progression to severe AKI requires a study design that can adequately address the question and appropriate sample size for each cohort as well as appropriate sample preparation and fractionation techniques and adequate sensitivity, resolution, and scan speed of the mass spectrometer, so that sufficient numbers of proteins can be seen with high enough sequence coverage to have confidence in the results. If these issues are adequately addressed, proteomic analysis is likely to identify novel biomarkers that can predict progression to severe AKI. Importantly, robust validation of biomarkers after the discovery phase is necessary for the biomarkers to be qualified for clinical use. 


\section{Acknowledgments}

Sources of support that require acknowledgement: NIH grant numbers R01DK080234, R01DK101034, P20GM121293, and UAMS Clinician-Scientist Program.

\section{Disclosure Statement}

The authors have no conflicts of interest to declare.

\section{References}

1 Koyner JL, Garg AX, Coca SG, Sint K, Thiessen-Philbrook H, Patel UD, Shlipak MG, Parikh CR; TRIBE-AKI Consortium: Biomarkers predict progression of acute kidney injury after cardiac surgery. J Am Soc Nephrol 2012;23:905-914.

2 Koyner JL, Davison DL, Brasha-Mitchell E, Chalikonda DM, Arthur JM, Shaw AD, Tumlin JA, Trevino SA, Bennett MR, Kimmel PL, Seneff MG, Chawla LS: Furosemide stress test and biomarkers for the prediction of AKI severity. J Am Soc Nephrol 2015;26:2023-2031.

3 Mishra J, Ma Q, Prada A, Mitsnefes M, Zahedi K, Yang J, Barasch J, Devarajan P: Identification of neutrophil gelatinase-associated lipocalin as a novel early urinary biomarker for ischemic renal injury. J Am Soc Nephrol 2003;14:2534-2543.
4 Supavekin S, Zhang W, Kucherlapati R, Kaskel FJ, Moore LC, Devarajan P: Differential gene expression following early renal ischemia/reperfusion. Kidney Int 2003;63:17141724.

5 Kashani K, Al-Khafaji A, Ardiles T, Artigas A, Bagshaw SM, Bell M, Bihorac A, Birkhahn R, Cely CM, Chawla LS, Davison DL, Feldkamp T, Forni LG, Gong MN, Gunnerson KJ, Haase M, Hackett J, Honore PM, Hoste EA, Joannes-Boyau O, Joannidis M, Kim P, Koyner JL, Laskowitz DT, Lissauer ME, Marx G, McCullough PA, Mullaney S, Ostermann M, Rimmele T, Shapiro NI, Shaw AD, Shi J, Sprague AM, Vincent JL, Vinsonneau C, Wagner L, Walker MG, Wilkerson RG, Zacharowski K, Kellum JA: Discovery and validation of cell cycle arrest biomarkers in human acute kidney injury. Crit Care 2013; 17:R25

6 Eliuk S, Makarov A: Evolution of orbitrap mass spectrometry instrumentation. Annual Rev Anal Chem 2015;8:61-80.

7 Wisniewski JR, Zougman A, Nagaraj N, Mann M: Universal sample preparation method for proteome analysis. Nat Methods 2009;6:359-362.

8 Yang F, Shen Y, Camp DG 2nd, Smith RD: High-pH reversed-phase chromatography with fraction concatenation for $2 \mathrm{D}$ proteomic analysis. Expert Rev Proteomics 2012;9:129-134.

9 Alge JL, Karakala N, Neely BA, Janech MG, Tumlin JA, Chawla LS, Shaw AD, Arthur JM; SAKInet Investigators: Urinary angiotensinogen and risk of severe AKI. Clin J Am Soc Nephrol 2013;8:184-193. 